\title{
Percepções e práticas profissionais no cuidado da obesidade na estratégia saúde da família
}

\author{
Perceptions and professional health care practices on obesity in the health family strategy
}

Amanda Tayná Tavares de Figueiredo ${ }^{a}$

Orcid: https://orcid.org/0000-0003-1439-5069

Fernanda Cristina de Lima Pinto Tavares ${ }^{b}$

Orcid: https://orcid.org/0000-0003-2118-7268

Priscila Ribeiro Rezende Mergulhão Silveira ${ }^{c}$

Orcid: https://orcid.org/0000-0001-7674-5613

\author{
Emilia Chagas Costa ${ }^{\mathrm{d}}$ \\ Orcid: https://orcid.org/0000-0002-7664-5994
}

Adrielly Araújo de Oliveira ${ }^{\mathrm{e}}$

Orcid: https://orcid.org/0000-0001-5940-1923

Pedro Israel Cabral de Lira ${ }^{f}$

Orcid: https://orcid.org/0000-0002-1534-1620

\begin{abstract}
Resumo
Introdução: A obesidade é uma epidemia global em ascensão no Brasil. A Estratégia Saúde da Família é a forma prioritária de organização da atenção básica, representando a principal porta de entrada e ordenadora do cuidado no Sistema Único de Saúde. Objetivos: Identificar concepções e práticas de cuidado ofertadas a pessoas com obesidade na Estratégia Saúde da Família. Materiais e Métodos: Estudo exploratório-descritivo, de método qualitativo, realizado em Recife-PE. Os dados foram coletados de dezembro de 2017 a fevereiro 2018, por entrevistas semiestruturadas com 12 profissionais de uma unidade de saúde, transcritas e submetidas à análise de conteúdo por categorias temáticas. $\mathrm{Na}$ análise consideraram-se 3 categorias definidas a priori (I-Formação Profissional; II-Conhecimento Prévio e; III-O Cuidado diante da Obesidade) e 8 subcategorias emergentes. Resultados: A obesidade foi concebida pelas profissionais como fenômeno multicausal, destacando a alimentação inadequada, o sedentarismo e os aspectos psicossociais em sua causalidade. Predominou práticas individuais com orientações sobre alimentação saudável, atividade física e autocuidado. Os usuários com obesidade foram vistos negativamente como passivos e desinteressados no cuidado. As fragilidades abrangeram a ausência de educação permanente sobre obesidade e dificuldade de integração com a rede especializada e a gestão. Conclusões: Embora as profissionais reconheçam a importância do cuidado integral, interdisciplinar, contínuo e em rede, identificaram-se desafios em operacionalizar tal entendimento. As práticas profissionais destinadas ao enfrentamento da obesidade na Estratégia Saúde da Família mostram a não superação do modelo assistencial biomédico com fragilidades relacionadas à educação permanente em saúde, ao vínculo profissional-usuário e à prática interdisciplinar e intersetorial.
\end{abstract}

Palavras-chave: obesidade; atenção primária à saúde; conhecimento, atitudes e prática em saúde; assistência integral à saúde; educação em saúde; segurança alimentar e nutricional.

\footnotetext{
a Mestranda da Pós-graduação em Nutrição na Universidade Federal de Pernambuco (PPGNUT/UFPE), Brasil. E-mail: figueiredo.att@gmail.com

b Universidade Federal de Pernambuco. Av. Nelson Chaves, Cidade Universitária, 50670-901, Recife, PE, Brasil. E-mail: fernanda_nutri2000@yahoo.com.br

${ }^{c}$ Programa de Residência Multiprofissional em Atenção Básica, Instituto de Medicina Integral Professor Fernando Figueira, Recife, PE, Brasil. E-mail: primergulhao@hotmail.com

${ }^{\mathrm{d}}$ Centro Acadêmico de Vitória, Universidade Federal de Pernambuco. Vitória de Santo Antão, PE, Brasil. E-mail: eccosta@hotmail.com

e Mestranda da Pós-graduação em Nutrição na Universidade Federal de Pernambuco (PPGNUT/UFPE), Recife (PE), Brasil. E-mail: adri.oliveira.nutri@gmail.com

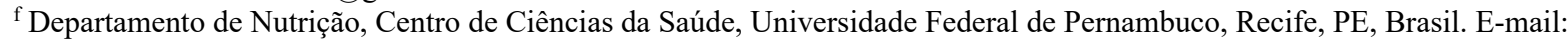
lirapic@ufpe.br
} 


\begin{abstract}
Introduction: Obesity is a rising global epidemic in Brazil. The Family Health Strategy is the priority model for primary health care representing the main gateway to care and coordinator role in the Brazilian Unified Health System. Objective: To identify understandings, perceptions and care practices related to obesity on a Family Health Strategy center. Materials and Methods: Exploratory-descriptive study of qualitative method. The study was placed in the city of Recife, Pernambuco, Brazil. Data were collected from November 2017 to February 2018, by semi-structured interviews with 12 professionals in a Family Health Strategy center. The audio were transcribed and analyzed by thematic categories. In the analysis were considered 3 categories defined a priori (I-Professional Training, II-Prior Knowledge and; IIICare in Obesity) and 8 emerging subcategories. Results: The professionals defined obesity as a multiple caused issue, emphasizing the role of unhealthy eating habits, physical inactivity and the psychosocial aspects on that. The dominant practices comprised counselling on healthy eating and exercise practicing, as well on selfcare. Obese individuals were negatively referred as passive and careless towards health care. Conclusions: Although the conceptions reinforce the comprehensive, interdisciplinary and networked care, there were challenges to apply these understandings. Professional practices for addressing obesity in the health family strategy reveal the non-overcoming of the biomedical care assistance model, suggesting the need for addressing weaknesses on continuing health education, the professional-obese patient relationship and interdisciplinary and intersectoral collaboration practices.

Keywords: obesity; primary health care; health knowledge, attitudes, practice; comprehensive health care; health education; food and nutrition security.
\end{abstract}

\section{Introdução}

Nas últimas três décadas o Brasil segue um processo de transição nutricional e epidemiológica, com crescimento da obesidade e aumento da morbimortalidade por doenças crônicas não transmissíveis. $\mathrm{O}$ excesso de peso está presente em 55,7\% da população adulta das capitais brasileiras, com $19,8 \%$ de obesidade, evidenciando uma evolução temporal desfavorável e significativa desses indicadores. Nesse panorama epidemiológico, a obesidade se destaca por ser simultaneamente uma doença e um fator de risco para outros agravos de saúde. ${ }^{1}$

Há consenso na literatura quanto ao caráter múltiplo e heterogêneo da obesidade com condicionantes genéticos, fisiológicos, comportamentais, ambientais, psicológicos, socioeconômicos, políticos e históricos. ${ }^{2}$ As complicações da obesidade para o indivíduo continuam sendo amplamente estudadas e são associadas a um elevado risco de desenvolvimento de diabetes mellitus, hipertensão, doenças cardiovasculares, dislipidemias, osteoartrite, diferentes tipos de câncer e dificuldades respiratórias e locomotoras. ${ }^{3,4}$ Além disso, pode comprometer a saúde mental por envolver aspectos psicológicos como controle, percepção de si, ansiedade e desenvolvimento emocional. ${ }^{5}$

No Brasil, o Ministério da Saúde é o principal propositor de ações mediante políticas públicas e documentos que abordam o enfrentamento da obesidade no âmbito do Sistema Único de Saúde (SUS). Nessa perspectiva, a Política Nacional de Alimentação e Nutrição, (PNAN) revisada em 2012, direciona ações de prevenção e tratamento da obesidade no SUS ${ }^{6}$. Em 2013 foram redefinidas as diretrizes para a organização da prevenção e do tratamento do sobrepeso e obesidade como linha de cuidado prioritária da Rede de Atenção à Saúde (RAS) das Pessoas com Doenças Crônicas. ${ }^{7}$ As publicações supracitadas reafirmam o papel da atenção primária como porta de entrada preferencial para a RAS, exercendo a ordenação e coordenação do cuidado, já previsto na Política Nacional de Atenção Básica (PNAB) ${ }^{8}$.

A atenção básica compreende ações de saúde individuais, familiares e coletivas relacionadas à prevenção, proteção, diagnóstico, tratamento, reabilitação, redução de danos, cuidados paliativos e vigilância em saúde, conduzidas por práticas de cuidado integrado e gestão 
qualificada, dirigidas à população em território definido. Nesse sentido, a Estratégia Saúde da Família (ESF) é a forma prioritária para expansão, qualificação e organização da atenção básica, dispondo de uma equipe multiprofissional denominada de equipe de Saúde da Família, composta por profissional médico, enfermeiro, técnico de enfermagem e agentes comunitários de saúde. ${ }^{8}$

As possibilidades de ações para o enfrentamento da obesidade na ESF estão relacionadas ao ciclo de gestão e produção do cuidado da Vigilância Alimentar e Nutricional (VAN), visando diagnóstico de agravos alimentares e nutricionais das populações, uma vez que o Sistema de Vigilância Alimentar e Nutricional é operado na atenção primária à saúde. ${ }^{9}$ No cenário de orientação de práticas destacamse o Caderno de Atenção Básica $n^{0}$ 38, Estratégias para o Cuidado da Pessoa com Doença Crônica Obesidade ${ }^{4}$, e o Marco de Referência da Vigilância Alimentar e Nutricional na Atenção Básica ${ }^{9}$, com diretrizes a respeito da linha de cuidado e qualificação da atenção destinada às pessoas com excesso de peso, no intuito de apoiar profissionais e gestores na organização da VAN.

A contínua ascensão da obesidade aponta para necessidade de reconhecer os desafios no enfrentamento desse problema dialogando com a ESF, a qual representa um espaço primordial para o exercício do cuidado integral e contínuo à população. Diante do exposto, o estudo objetivou identificar as concepções e práticas de cuidado ofertadas a pessoas com obesidade na ESF do território de uma Unidade de Saúde da Família (USF) da cidade do Recife.

\section{Métodos}

Trata-se de um estudo exploratóriodescritivo, de método qualitativo, realizado em uma USF na cidade do Recife vinculada ao Programa de Residência Multiprofissional em Atenção Básica pelo
Instituto de Medicina Integral Prof Fernando Figueira. O presente estudo integrou as atividades do programa de residência supracitado. A seleção da amostra foi intencional. Os participantes foram profissionais de diferentes categorias, das duas equipes de saúde da família e da equipe de saúde bucal atuantes na USF selecionada.

A coleta de dados foi realizada entre dezembro de 2017 e fevereiro de 2018, na USF, por meio de entrevistas semiestruturadas, conduzidas individualmente, gravadas em áudio digital, com roteiro de perguntas abertas distribuídas em três categorias temáticas definidas a priori: formação profissional, conhecimento prévio e cuidados à pessoa com obesidade. As categorias, a priori, correspondem a segmentos de ampla abrangência contemplados no referencial teórico da investigação, definidos previamente para acolher as subcategorias emergentes a serem identificadas na análise 10 .

A construção do roteiro da entrevista se deu a partir da definição das categorias apriorísticas, processo no qual foi considerada a influência das experiências, do conhecimento dos indivíduos e das relações humanas nas práticas profissionais enquanto elementos estruturantes do trabalho em saúde na perspectiva da racionalidade da práxis como lógica mediadora entre o saber e o fazer ${ }^{11}$.

Para elaborar as perguntas dirigidas às práticas, considerou-se a organização da atenção à saúde prevista no Caderno de Atenção Básica n. 38, no qual abordam-se estratégias para o cuidado da pessoa com obesidade envolvendo a educação permanente aos profissionais, o conceito ampliado de saúde, as relações entre as equipes, usuários e famílias, a dinâmica da comunidade e as políticas públicas ${ }^{4}$. Além disso, a construção do instrumento teve por referência o guia das entrevistas semiestruturadas aplicado em um cenário de pesquisa com profissionais da atenção 
primária em Portugal para investigar suas crenças, atitudes e práticas na obesidade ${ }^{12}$.

O material gravado foi transcrito na íntegra e submetido à análise de conteúdo segundo Bardin ${ }^{13}$, desenvolvida nas etapas de pré-análise, exploração do material, tratamento dos resultados e interpretação. Seguindo as etapas, foi realizada leitura flutuante e releituras para assimilar de forma global e não sistemática as principais ideias e os significados gerais. Em seguida, ocorreu seleção das unidades de análise, para as quais foram atribuídos códigos descritivos. No processo inicial de codificação, os códigos foram agrupados por correspondência com as sessões do roteiro da entrevista referentes às categorias definidas a priori.

Posteriormente, em cada categoria principal, os códigos foram reagrupados por proximidade e diferenciação em função dos seus significados, considerando a recorrência e relevância implícita dos mesmos $^{10}$ para a construção das subcategorias. $\mathrm{O}$ tratamento dos dados consistiu na interpretação e inferência sobre os resultados sistematizados nas subcategorias emergentes, a partir dos pressupostos teóricos do presente estudo.

Os resultados e discussão foram organizados nas categorias principais definidas a priori e suas respectivas subcategorias emergentes, identificadas durante a análise de conteúdo. Para cada participante foi atribuído um código com o intuito de preservar a identidade dos mesmos na apresentação dos resultados. Uma única entrevistadora conduziu as entrevistas, tratando-se de nutricionista, devidamente treinada e integrante de uma equipe multidisciplinar de residentes vinculada à USF em questão.

Esse estudo foi aprovado pelo Comitê de Ética e Pesquisa em Seres Humanos do Instituto de Medicina Integral Prof Fernando Figueira (IMIP), sob o parecer de número 2.371.361. Os participantes foram convidados a participar da pesquisa e esclarecidos sobre a entrevista a ser realizada e sigilo das informações concedidas durante a mesma. Após os esclarecimentos, os convidados que consentiram participar assinaram um Termo de Consentimento Livre e Esclarecido.

\section{Resultados e discussão}

Foram realizadas 12 entrevistas com profissionais da USF selecionada, identificados pelos códigos $\mathrm{P} 01$ a P12 e sua respectiva categoria profissional, com duração variável de 8 a 25 minutos. Para melhor compreensão dos dados observados, ressalta-se que os resultados são referentes ao cotidiano de uma USF, não sendo, portanto, um grupo especializado em obesidade.

As 3 temáticas gerais e 8 subcategorias emergentes foram: Formação Profissional - Perfil dos Profissionais e Educação Permanente em Saúde; Conhecimento Prévio - Reconhecendo a Obesidade, Implicações da obesidade para o indivíduo e Como Tratar a Obesidade; e O Cuidado diante da Obesidade - Práticas de Cuidado na USF e Território, Percebendo o Usuário com Obesidade no cuidar, e O papel da ESF e da RAS no Enfrentamento da Obesidade.

\section{Formação Profissional}

\section{Perfil dos Profissionais}

As participantes eram do sexo feminino, com idade entre 28 e 64 anos, a maioria acima de 40 anos (75\%), com escolaridade de nível médio, técnico e superior, compreendendo 5 agentes comunitárias de saúde, 2 enfermeiras, 2 médicas, 1 técnico de enfermagem, 1 cirurgiã dentista e 1 auxiliar de saúde bucal. O tempo de atuação na atenção básica variou de 4 a quase 30 anos, e a maioria atuava há mais de 15 anos naquela unidade de saúde. Apesar de haver variação na forma de ingresso no processo histórico de estruturação do serviço, todas as 
entrevistadas eram servidoras públicas efetivadas à época da coleta de dados.

Pelo tempo de atuação das entrevistadas na unidade, a maioria vivenciou importantes reformulações da assistência ofertada no território, como ações de saúde da Pastoral da Criança, na qual já havia a figura do agente comunitário, e implantação do Programa de Agentes Comunitárias de Saúde e do Programa de Saúde da Família. Assim, algumas profissionais começaram a atuar na localidade da USF vinculadas a instituições filantrópicas e, posteriormente, foram integradas às equipes de saúde reguladas pelo SUS e gestão municipal.

\section{Educação Permanente em Saúde}

Educação Permanente pode ser compreendida por processos de aprendizagem direcionados a partir do cotidiano dos profissionais e das demandas de saúde individuais e coletivas, com potencial de qualificar e organizar os processos de trabalho desenvolvidos na atenção ${ }^{4}$ No entanto, nenhuma das profissionais participou de formação sobre obesidade como tema principal. Algumas participaram de ações pontuais e expositivas que envolviam obesidade como tema secundário, as quais não foram consideradas adequadas pelas entrevistadas para orientar práticas voltadas à obesidade.

"Não. Sempre falam os agravos que a obesidade causa, né, mas em questão só sobre aquele tema 'o que é obesidade', não”. (P09, Agente Comunitária de Saúde)

Os relatos evidenciam que a obesidade não foi objeto das ações de educação permanente ofertadas às equipes representadas pelas entrevistadas, indicando que elas ainda não vivenciaram de forma sistemática a problematização do processo de trabalho diante da epidemia da obesidade. Ao investigar estratégias de educação permanente em alimentação e nutrição na ESF de municípios brasileiros de grande porte, Ricardi e Sousa (2015) verificaram que a não priorização desse tema e a insuficiência de profissionais nutricionistas foram dificultadores para a realização de tais estratégias ${ }^{14}$.

Tendo em vista o trabalho interdisciplinar, construído no exercício cotidiano por profissionais de diferentes categorias ao integrar e aplicar seus núcleos de conhecimento em um único campo de atuação ${ }^{15}$, a inserção de categorias não contempladas nas equipes de saúde da família nos processos de educação permanente em saúde, tais como nutricionistas, profissionais de educação física e psicólogos, representa uma condição estratégica para a qualificação das equipes.

Assim, a educação permanente permite ampliar a resolutividade da prevenção e assistência aos casos de obesidade e suas comorbidades, tanto por meio de ações educativas destinadas ao enfrentamento da obesidade, quanto pelo compartilhamento de saberes e práticas na assistência aos usuários. Tal construção interdisciplinar pode ser efetivada com apoio matricial, no qual objetiva-se implementar e fortalecer espaços de comunicação ativa para a integração de saberes entre os profissionais envolvidos na produção do cuidado em questão ${ }^{16}$.

As entrevistadas afirmaram não conhecer nenhum material sobre obesidade, o que revela então o desconhecimento e, consequentemente, não utilização das publicações do Ministério da Saúde desenvolvidas para apoiar os profissionais da atenção básica na prevenção e tratamento do excesso de peso, tais como o Caderno de Atenção Básica n. $38^{4}$ e o Marco de Referência da Vigilância Alimentar e Nutricional na Atenção Básica ${ }^{9}$.

Quanto ao desconhecimento de documentos norteadores das práticas profissionais para o enfrentamento da obesidade na atenção básica e em outros níveis da RAS, esse cenário também foi identificado na atenção nutricional ofertada em unidades básicas de saúde em um município da região metropolitana de Teresina, estado do Piauí, não se tratando de 
uma fragilidade limitada à realidade do presente estudo ${ }^{17}$.

Tal situação reflete a importância da articulação e planejamento dos setores de educação permanente dos três níveis de gestão (federal, estadual e municipal), não só para a elaboração de instrumentos adequados, mas sobretudo para a divulgação, sensibilização, inserção e utilização efetiva desses pelos profissionais que têm o acesso direto à população.

\section{Conhecimento Prévio}

\section{Reconhecendo a Obesidade}

A maioria das participantes associou obesidade a valores elevados de uma ou mais medidas antropométricas, como peso, circunferência da cintura, índice de massa corporal e relação entre peso e estatura; três relacionaram ao excesso de peso e/ou gordura corporal que compromete a saúde do indivíduo e duas utilizaram os elementos de aceitação do peso corporal, autocuidado, alterações metabólicas e hábitos alimentares para definir a obesidade.

"Obesidade eu acho que é aquela pessoa que se sente mal com o seu peso". (P05, Agente Comunitária de Saúde)

"É uma síndrome onde a pessoa tem um excesso de peso e esse excesso de peso traz consigo as doenças né, as dislipidemias, a diabetes, a hipertensão, as doenças articulares, as doenças mentais também, os problemas psíquicos né, de baixa autoestima. Enfim, é uma sindrome bem complexa". (P11, Médica)

Logo, não houve homogeneidade nas definições de obesidade, uma minoria se aproximou do conceito da Organização Mundial de Saúde, em que a obesidade é um agravo multifatorial decorrente de um balanço energético positivo que leva ao acúmulo excessivo de gordura corporal e comprometimento da saúde dos indivíduos ${ }^{3}$.

Todas as participantes apontaram mais de uma causa para a obesidade, entre má alimentação, sedentarismo, prédisposição genética, patologias de base, a família, o estado psicológico e emocional e o gasto calórico reduzido.

"Tem o fator de genética, mas acredito que o fator psicológico é muito forte... na minha família tem um caso assim que vem muito porque foi abusada, então depois disso começou com essa questão da ansiedade por comida, a compulsividade". (P01, Agente Comunitária de Saúde)

"É a má alimentação, a falta de exercício fisico”. (P02, Enfermeira)

Dado o consenso quanto ao caráter múltiplo e heterogêneo da obesidade, as profissionais entendem que não há causalidade isolada e linear, mas uma interação complexa entre variáveis biopsicossociais. Embora o entendimento seja adequado, pode ser considerado incompleto se não existir associação das causas citadas com a influência do ambiente em que os indivíduos estão inseridos ${ }^{2}$. Portanto, fatores ambientais devem ser observados na compreensão da etiologia e na construção de propostas para prevenção e tratamento da obesidade na ESF. Nesse sentido, verifica-se a necessidade de incorporação de uma perspectiva sistêmica $^{18}$ e sindêmica à compreensão e propostas de intervenções efetivas diante da obesidade $^{19}$, que embora manifeste-se com singularidades locais, apresenta casualidades globais.

\section{Implicações da obesidade para o indivíduo}

As profissionais apresentaram respostas semelhantes sobre as consequências da obesidade para o indivíduo. A maioria citou exemplos de implicações metabólicas, osteomusculares, psicológicas e sociais, uma se deteve aos impactos psicossociais e duas expressaram que existiam muitas consequências sem citar exemplos.

Ao abranger agravos de saúde e implicações psicossociais, as profissionais revelaram estar cientes das variadas 
demandas que podem surgir nos casos de obesidade, favorecendo a integralidade e efetivação do cuidado centrado na pessoa, diretriz operativa da RAS que consiste em ações de cuidado singularizadas, construídas com as pessoas, de acordo com suas necessidades e potencialidades ${ }^{7}$.

\section{Como Tratar a Obesidade \\ Os aspectos}

mencionados pelas participantes envolveram o cuidado ofertado nas unidades de saúde, a atitude da pessoa com obesidade diante do seu diagnóstico, a atitude dos profissionais e a participação da família. Foi frequente a observação de que era preciso um cuidado multiprofissional e acesso a especialistas. Quanto à atitude da pessoa com obesidade, expressaram que o tratamento demanda a aceitação do problema, determinação, estímulo pessoal e mudanças no estilo de vida, principalmente reeducação alimentar e prática de atividade física.

\begin{abstract}
"De forma multiprofissional. O médico está aqui para fazer o diagnóstico, orientar, mas tem que ter a nutrição para orientar a questão da alimentação. Tem que ter o educador físico para orientar. Inclusive porque o obeso não pode fazer qualquer atividade física, nem pode iniciar de uma forma mais pesada. Tem a questão da psicóloga também que faz com que ele se aceite melhor, enfim”. (P11, Médica)

"Eu acredito que inicialmente tem que haver uma reeducação, uma mudança de estilo de vida, ai focalizando a redução da ingestão de açúcares (...) tem o socioeconômico, e muita ingestão de carboidratos. É, o combate ao sedentarismo e a questão de lazer, procurar reduzir a ansiedade e por ai vai... A própria escolaridade reduziria bastante a obesidade porque ai você teria uma concepção e compreensão melhor disso tudo que a gente está falando aqui”. (P12, Médica)
\end{abstract}

Algumas participantes enfatizaram que orientações dadas por profissionais sobre alimentação precisam ser compatíveis com a condição socioeconômica dos usuários, considerando o poder aquisitivo e consequentemente o acesso aos alimentos. A participação da família foi apontada como apoio à adesão ao tratamento da obesidade. Foram citadas por uma única vez as abordagens com uso de medicações, intervenção cirúrgica, combate à ansiedade e estímulo ao lazer. A menção à influência da escolaridade no tratamento do excesso de peso é consoante aos resultados observados no Vigitel (2019), em que a prevalência excesso de peso entre mulheres adultas é significativamente maior nos estratos de menor escolaridade ${ }^{1}$.

As possibilidades de tratamento sugeridas estão inseridas em uma perspectiva ampla de cuidado que valoriza a composição multidisciplinar das equipes de saúde da família, a necessidade de articulação entre os diferentes pontos da RAS, e a relevância de aspectos subjetivos como a atitude dos usuários, dos profissionais e o apoio familiar para resolutividade do cuidado. Os conhecimentos referidos pelas profissionais apresentam concordância com as observações de Palmeira et al. (2016), em que a percepção de pessoas sobre sua condição de obesidade contemplava significados para além do peso corporal e de agravos físicos, alcançando o reconhecimento de comprometimentos sociais, profissionais e psicológicos, com insatisfação de imagem corporal, declínio de autoestima, isolamento e exclusão social. Ainda, observou-se que tais indivíduos referiram buscar o tratamento da obesidade por meio de reeducação alimentar e atividade física, admitindo ter dificuldades de seguir essas estratégias a longo prazo e o desejo de serem acompanhados por diferentes profissionais ${ }^{20}$.

\section{Cuidados à Pessoa com Obesidade}

\section{Práticas de Cuidado na USF e território}

Para compreender a rotina das entrevistadas envolvendo usuários com obesidade, questionou-se a frequência de atendimento a esse público e a oferta de 
cuidado. Desse modo, a maioria das profissionais afirmou que lidava com usuários obesos diariamente e uma delas enfatizou o aumento dos casos. Duas relataram acolher pessoas com obesidade regularmente sem detalhar a frequência e uma não respondeu. Duas agentes comunitárias de saúde verbalizaram que não lidavam com usuários obesos rotineiramente devido às características específicas de suas microáreas de atuação. Uma explicou que não havia muitos casos em sua microárea, portanto seria adequado um trabalho preventivo, a outra conhecia os casos de obesidade de sua microárea, mas sabia que essas pessoas não frequentavam a USF.

"Eita! Frequentemente viu? Bastante. Eu tenho visto que tem aumentado muito (...) todo dia aparece um aqui na minha sala”. (P08, Dentista)

"Acontece uma vez ou outra quando eu encontro com a pessoa, porque tem pessoas que eu não vejo diariamente. Agora tô querendo ir nessas casas dessas pessoas que eu vejo que são obesas e não vejo muito aqui no posto". (P09, Agente Comunitária de Saúde)

As observações das profissionais sobre o aumento dos casos de obesidade na população adscrita à USF refletem o panorama do município e do país, considerando que em 2016 a prevalência de obesidade entre a população maior de 18 anos no Recife foi de $20 \%$, com crescimento de $60 \%$ no Brasil em uma década $^{21}$.

O processo de trabalho territorializado possibilita vínculos para além das rotinas dos consultórios e desenvolvimento de ações coletivas, de prevenção e potentes práticas para redução de taxas de prevalência da obesidade em médio e longo prazo.

As práticas de cuidado descritas com recorrência consistiram em realizar perguntas para conhecer os hábitos alimentares dos usuários, orientar de forma geral sobre alimentação saudável e prática de atividade física, sensibilizar quanto à importância de cuidar da alimentação, de uma rotina ativa e de idas regulares à USF para acompanhamento médico $\mathrm{e}$ continuidade do cuidado.

Entre as agentes comunitárias de saúde, que circulam no território com maior frequência, é comum que observem quais alimentos são encontrados nos domicílios durante as visitas. As profissionais de nível técnico não realizam orientações sobre alimentação e atividade física, uma delas relatou que ao identificar uma pessoa com obesidade costuma encaminhar para equipe multidisciplinar de residentes ou para as médicas da USF.

A maioria das profissionais de nível superior expressou realizar a sensibilização para os cuidados com a saúde, não havendo um padrão nas práticas desse grupo, abrangendo as seguintes ações: realização de orientação alimentar mais detalhada; uso de roteiro próprio para abordar alimentação saudável, sedentarismo, lazer e combate à ansiedade; solicitação imediata de exames bioquímicos para auxiliar o usuário a reconhecer os agravos à saúde decorrentes do excesso de peso; verificação do desejo do usuário em ser encaminhado; e encaminhamentos à rede especializada.

"É, dou orientação, no momento pergunto do que ela se alimenta, do que tem em casa pra se alimentar e até passo umas receitas pra que ela mude um pouco o estilo de alimentação". (P09, Agente Comunitária de Saúde)

"A primeira coisa que eu oferto são os exames. E até para ela enxergar o problema da obesidade, porque às vezes a pessoa não está nem aí, é gorda e o problema da obesidade é só estética, ela não entende que o problema da obesidade está fazendo um mal muito maior para ela, né?!” (P11, Médica)

"Durante os atendimentos eu busco encaminhar. Ofereço primeiro (o encaminhamento), porque às vezes você está vendo a situação do paciente, mas ele não está aceitando (...) você sensibiliza ele e diz que se precisar e quando quiser pode vir. E aqueles que já vem de imediato, a gente solicita os encaminhamentos para as especialidades. A pena é que, assim, às 
vezes ou em uma grande parte, esses encaminhamentos demoram para dar uma resposta ao paciente e ai a vida passa e eles seguem adiante”. (P03, Enfermeira)

Observa-se a intenção por parte das profissionais em ofertar cuidados compatíveis ao cotidiano dos usuários com obesidade. No entanto, na prática, predomina a abordagem com a obesidade como fenômeno de causa individual, dissociando as orientações e soluções do ambiente familiar e comunitário, prevalecendo orientações prescritivas $\mathrm{e}$ genéricas.

Resultados semelhantes foram observados por Gomes et al. (2017) ao analisarem as orientações alimentares ofertadas por profissionais de saúde de nível superior da ESF a usuários hipertensos e/ou diabéticos $^{22}$. Portanto, embora as profissionais entrevistadas no presente estudo reconheçam a necessidade da abordagem multidisciplinar e centrada na pessoa, o cuidado ofertado predominantemente não corresponde ao cuidado idealizado.

"Algumas coisas a gente tem noção né, de orientar, 'mas por que tu não faz uma caminhada?!', na questão da redução de peso. Me meto também na questão da alimentação, porque tem gente que quer, mas parece que só quer da boca pra fora, né”? (P01, Agente Comunitária de Saúde)

O empenho coletivo das profissionais em sensibilizar usuários com obesidade para irem até a USF potencializa o papel da atenção básica como porta de entrada preferencial da RAS. Da mesma forma, a coordenação do cuidado é fortalecida mediante os encaminhamentos realizados pelas profissionais de nível superior, as quais relataram referenciar usuários obesos aos laboratórios e componente ambulatorial da rede de acordo com suas demandas.

A prática das agentes comunitárias ao observar o acesso a alimentos durante as visitas domiciliares destaca a importância do trabalho territorializado da atenção primária, para se manter um olhar contínuo sobre a situação da segurança alimentar e nutricional das famílias e indivíduos ${ }^{7,9}$, uma vez que a obesidade é uma das manifestações da insegurança alimentar e nutricional que acomete a população brasileira. Nessa perspectiva, a partir da análise de estudos sobre estratégias utilizadas na gestão da atenção primária à saúde para controle da obesidade, Almeida et al. (2017) apontaram desafios relacionados ao papel dos agentes comunitários enquanto promotores de saúde e insuficiência de práticas contextualizadas às condições de vulnerabilidade social da população ${ }^{23}$.

Diante do conjunto de práticas identificadas, apesar de constituírem atividades de assistência terapêutica e de vigilância, há escassez de ações contínuas, que integrem os diferentes profissionais e usuários de forma colaborativa e que explorem o território como cenário de prática. Apresentando práticas e desafios comuns aos supracitados, a orientação alimentar e de atividade física foram consideradas como as principais ações da atenção nutricional ofertada a usuários com obesidade na ESF, apontadas por profissionais e gestores de um município da região metropolitana da capital do Piauí, destacando fragilidades na integralidade de tal cuidado em decorrência da infraestrutura inadequada, dificuldades com recursos humanos, falta de nutricionista e necessidade de maior apoio da gestão local 17.

A identificação de problemas e soluções locais é essencial para ampliar a efetividade das práticas de cuidado, a autonomia dos usuários e a capacidade de mudança no estilo de vida na dinâmica territorial em que estão inseridos. Uma minoria entre as entrevistadas identificou fatores limitantes e propôs alternativas relacionadas a determinantes além dos intrínsecos aos indivíduos. Foi exposta a falta de espaços para realização de exercícios e atividades grupais, insegurança nas ruas, dificuldade de acesso a alimentos 
in natura e a calçados adequados para atividade física na comunidade.

\begin{abstract}
"Às vezes já ouvi dizer 'eu não tenho tênis, não tenho condições de comprar um tênis, não tenho tempo de ir caminhar' (...) A gente sempre tenta fazer com que ele faça alguma coisa para melhorar a sua situação (...) aqui mesmo a gente não tem uma praça pra fazer uma caminhada (...) não tem o estímulo dos nossos governantes, eles não estão nem aí para fazer com que ajude a unidade de saúde da família para fazer com que caia a obesidade, tanto de criança como adulto. Porque isso poderia ser melhorado com o quê? Vamos dizer, se eles colocassem uns ônibus com verdura, com peixe, mais barato que o pessoal pudesse comprar. Se tivesse, assim, uma vez na semana, aquele onibus em cada bairro ou em cada comunidade (...) um peixinho mais em conta, uma fruta mais em conta, sabe como é?! É porque assim, o que muitos se alimentam é do que tem no momento para comer (...) o que está mais faltando é a verdura deles (...) às vezes até também por conta do paciente com preguiça, eles querem o mais fácil, mas a gestão poderia assim, atuar mais, ver mais o lado da gente. Porque às vezes a gente quer seguir adiante, mas a gente para por causa da precariedade do nosso trabalho. Tu já pensasses assim, vamos dizer, tanto de alimento como um carro também com tênis! Tênis mais barato, podia até ser assim faltando, faltando um cadarço, ou um pouco descolado que desse para pessoa colar, a pessoa poderia comprar uma roupa, um tênis pra caminhar, né?! Para poder se estimular e ter uma autoestima melhor e poder se cuidar". (P09, Agente Comunitária de Saúde)

"Muita gente tava fazendo atividade física com um rapaz de educação física (comunitário), ai ele incentivou muita gente pra fazer caminhada, ai até que tava um número de pessoas até bom à noite, fazia aula à noite, mas só que é aquela coisa, violência! Essas coisas tudinho, né, ai muita gente desistiu”. (P10, Agente Comunitária de Saúde)
\end{abstract}

Percebe-se que ao identificar uma rede de apoio deficitária e, em alguns setores, inexistente, parte das entrevistadas manifestam sensação de impotência diante de casos de obesidade. A dificuldade para conciliar o entendimento da multideterminação da obesidade com as práticas de cuidado na USF e território reflete lacunas em educação permanente, gestão do trabalho, organização da RAS e subestimação do problema do excesso de peso, o qual é emergencial, epidêmico e transversal a todos os componentes da RAS e setores extra saúde. Assumindo a magnitude de tal problema, Lee BR et al. (2017) propõem um modelo de abordagem sistêmica para prevenção e controle da obesidade com fundamental articulação entre pesquisa, educação, políticas e ações ${ }^{18}$.

Percebendo o usuário com obesidade no cuidar

A fim de entender a relação das profissionais com os usuários obesos, investigaram-se as principais queixas e demandas desses usuários e como as profissionais se sentiam cuidando dessas pessoas. As queixas mais recorrentes foram dores osteomusculares, dificuldades respiratórias e de locomoção, e cansaço. Identificaram ainda, tristeza, limitações para realizar atividades da vida diária, constrangimento durante os atendimentos devido à falta de materiais adequados para obesos e demandas relacionadas à dislipidemia e esteatose hepática.

A maioria das profissionais expressou que os usuários obesos normalmente não buscam a USF por causa da obesidade, mas por respostas de curto prazo a agravos agudos e que muitos esperam ter acesso à nutricionista, endocrinologista e soluções rápidas para perda de peso e redução do apetite. A percepção geral entre as entrevistadas é que há um desinteresse por parte das pessoas com obesidade em iniciar processos de cuidado contínuo e com mudanças no estilo de vida.

"É muito relativo. Tem uns que realmente enxergam que estão bem acima do peso e que tem a vontade de 
reduzir o peso, mas tem outros que não, que só querem resolver aqueles problemas momentaneamente. Chega assim 'ah, estou com uma dor no joelho', ai a gente explica que é o excesso de peso também que sobrecarrega as articulações, mas às vezes ele não quer tratar a causa base que é a obesidade. Ele quer aliviar a dor dele. A resposta imediata. São poucos que querem e aceitam entrar na reeducação alimentar". ( $\mathrm{P} 02$, Enfermeira)

Duas

profissionais complementaram que apesar de não existir um interesse específico pelo tratamento da obesidade, as pessoas que se encontram obesas estão em busca de ajuda. Uma delas afirmou que os profissionais precisam obter e oferecer informações de qualidade, com atitude sensível e cuidadosa para não atribuir culpa às pessoas pela sua condição de obesidade.

"Não basta ter informação, é preciso ter oferta de mudanças na qualidade de vida. Porque assim, o que a gente tem visto muito é que a gente começa a culpar as pessoas 'você é culpada porque você bebe muito refrigerante, você come de forma inadequada, você não faz atividade física... você, você, você! 'e muitas vezes a gente não está vendo que aquela criatura é um sofrimento muito grande”. (P03, Enfermeira)

As percepções negativas e atenção à culpabilização dos usuários reafirmam a presença do estigma da obesidade nos espaços de produção de cuidado, com implicações negativas na saúde dos indivíduos com excesso de peso, uma vez que a estigmatização está associada simultaneamente ao agravamento da obesidade e à potencialização dos seus fatores de risco comportamentais. Embora evidente, discussões acerca do estigma da obesidade ainda são escassas na implementação de estratégias preventivas e de enfretamento dessa epidemia ${ }^{24}$. Nesse sentido, Rubino et al. (2020) destacaram a necessidade do fomento de iniciativas voltadas à prevenção da discriminação em decorrência do peso corporal em espaços profissionais, educacionais e de saúde ${ }^{25}$.

Apenas quatro profissionais expressaram sentimentos positivos associados ao cuidar na obesidade, partindo de interesse pessoal na temática, desejo de compartilhar o próprio relato de mudança de estilo de vida e sensação de contentamento ao se enxergar contribuindo para o cuidado. No entanto, a maior parte das profissionais sentia que era um processo difícil, atribuindo as dificuldades à atitude dos usuários, preconceito dos profissionais, ausência de capacitações sobre o tema, obstáculos para realizar procedimentos técnicos por falta de materiais adequados e limitações da rede de apoio. Entre as dificuldades citadas, a atitude dos usuários com obesidade se sobressaiu, sendo recorrente a percepção de que muitos não aceitam sua condição, não enxergam o excesso de peso como problema de saúde, não se interessam em mudar e apresentam baixa adesão a tratamentos.

"Não é fácil porque aquela história da capacitação é muito importante, eu sinto necessidade de ter essa capacitação, até para gente ter um olhar menos preconceituoso (...) a gente precisa ter esse olhar acessivel (...) ter uma oferta de maiores profissionais, de uma rede de apoio. A gente precisava ter alguém da academia da cidade também trabalhando com a gente (...) agora é aquele trabalho de formiguinha (...) intenso e contínuo e de muita sensibilização”. (P03, Enfermeira)

"Eu acho que é dificil (...) a pessoa tem que querer, tem que se ver, se olhar, 'eu tô precisando, porque além de estar obeso está comprometendo outra parte da minha saúde, não tá legal né’ (...) a pessoa tá com aquela situação mas não se enxerga, que está precisando de ajuda, ai eu acho dificil". (P07, Auxiliar de Saúde Bucal)

As falas indicam que a resolutividade do cuidado é atribuída principalmente à adesão e ao autocuidado dos usuários com obesidade, ainda que tenham sido consideradas as dificuldades estruturais, de fluxo pela rede e na educação 
permanente dos profissionais. $\mathrm{O}$ destaque à atitude dos obesos, dita como desinteressada e passiva, é consoante à percepção de profissionais da atenção primária à saúde em Portugal, com crenças e atitudes negativas em relação aos obesos, descritos como desmotivados e passivos diante dos tratamentos, não valorizando a obesidade enquanto problema de saúde ${ }^{12}$. No mesmo sentido, Sonntag et al. (2012) identificaram que médicos da atenção primária em Berlim percebiam o cuidado ofertado aos pacientes obesos como inefetivo e frustrante ${ }^{26}$.

Ainda no cenário de crenças e práticas voltadas à obesidade, uma revisão sistemática de treze artigos dos EUA, Reino Unido e Israel, observou que clínicos gerais e médicos da família consideravam difícil abordar a obesidade nos seus serviços, não demonstravam consistência no conhecimento acerca do tema e nas práticas, com ceticismo quanto à eficácia das intervenções existentes e percepção negativa sobre pacientes obesos, vistos como preguiçosos, desmotivados e sem autocontrole $^{27}$.

A fim de evitar um ambiente reducionista na ESF, é necessário ampliar a ideia de adesão e autocuidado para além do cumprimento de orientações, prescrições e assiduidade nos pontos de atenção ${ }^{28,29}$. Assim, não há margem para que somente a população seja culpabilizada por seus agravos de saúde, permitindo a construção de vínculos horizontais, corresponsabilização no cuidado, construção compartilhada de novas abordagens e reconhecimento das limitações dos profissionais.

\section{O papel da ESF e da RAS no enfrentamento da obesidade}

Somente duas participantes não responderam como a ESF contribui no enfrentamento da obesidade, as demais citaram potencialidades e/ou fragilidades baseadas em suas vivências de profissionais de saúde. Sobre as potencialidades, enfatizaram a prática rotineira de orientações gerais para mudanças no estilo de vida e os atendimentos clínicos realizados pelas médicas e enfermeiras da USF. Incluiu também o fácil acesso a profissionais médicos, o acolhimento que é realizado diariamente na USF, a coleta de dados de peso e altura para sistemas de informação em saúde, a proximidade das profissionais ao cotidiano dos usuários, a educação em saúde voltada para a prevenção, a escuta e os encaminhamentos à atenção especializada.

"O que a gente pode fazer a gente faz. Porque médico clínico geral resolve, agente de saúde dá conselho, a gente consegue ver a limitação, o momento da angústia dela, o que é que ela está sentindo ali, a gente escuta. A escuta é a melhor coisa, mas a gente faz o que pode". (P05, Agente Comunitário de Saúde)

"A questão das orientações, educação em saúde, eu acho que é a base de tudo. A gente começa desde a criança. Eu procuro orientar desde a criança, $e$ quando eu vou orientar a questão da educação alimentar para um adulto eu sempre digo 'Olhe, isso não é só pra você que é doente não, que está doente, isso é desde da criancinha, o pequenininho tem que ter esse cuidado, o adulto, o idoso, todos' A questão do excesso do açúcar, da gordura, do sal, que é uma constante aqui e eu sempre oriento, desde a infância isso é importante, né!?" (P11, Médica)

"Ah, contribui muito porque, primeiro tem o acesso direto ao usuário, né!? E depois pode encaminhar pra alguns setores. Apesar da demanda, ainda, da demora, é sempre do posto de saúde que se pode encaminhar né, para algum tipo de tratamento". (P06, Agente Comunitário de Saúde)

As potencialidades da ESF apontadas pelas profissionais são consoantes com parte das atribuições da atenção básica para prevenção e tratamento do sobrepeso e obesidade na RAS. Tais atribuições consistem na realização parcial da VAN mediante coleta de dados antropométricos e uso de sistemas de 
informação em saúde, desenvolvimento de ações de prevenção ao sobrepeso e obesidade, assistência terapêutica multiprofissional a indivíduos com excesso de peso, coordenação do cuidado de indivíduos quando necessitarem de possibilidades terapêuticas em outros pontos de atenção e garantia do acolhimento adequado das pessoas com sobrepeso e obesidade. ${ }^{7}$

A respeito das fragilidades, concordaram que a assistência encontrada na ESF era deficitária e insuficiente. Três profissionais especificaram dificuldades vinculadas ao processo de trabalho na USF e ao território, resgatando a falta de capacitações sobre obesidade, fragmentação e descontinuidade de práticas, e ausência de dispositivos públicos e sociais no território para realização de atividade física.

\begin{abstract}
"Deveria haver uma sistematização, e uma preocupação maior, mas ainda é muito descontinuado. Ainda é muito do envolvimento do profissional isolado, eu acho que precisa de mais sistematização do cuidado do obeso". (P12, Médica)

"Pode até ser que ela (ESF) contribua assim de orientação no consultório, viu!? (...) No coletivo, não! Pode ser no individual, no consultório, isolado, médico orientando a nutrição, mas assim, no enfrentamento do coletivo, eu ainda não presenciei isso não (...) como aqui na unidade a gente não tem nenhuma referência de uma academia da cidade, ai já vai metade do nosso programa para aquele obeso, porque ele tem que fazer uma atividade física (...) então tem que ter as parcerias (...) e parceria é complicado aqui”. (P08, Dentista)
\end{abstract}

A percepção das profissionais sobre o papel da ESF no enfrentamento da obesidade indica desafios na efetivação de um trabalho interdisciplinar e intersetorial. Farias et al. (2018) verificaram a existência de obstáculos para interdisciplinaridade no cotidiano de trabalhadores da ESF relacionando-as ao modelo das graduações em saúde, insuficiência de educação permanente voltada à interprofissionalidade, sobrecarga de trabalho e necessidade de iniciativas da gestão que viabilizem a integração entre os profissionais $^{30}$. Silva et al. (2014) observaram, em seis municípios brasileiros, que apesar de existir intenção e potencial de realizar ações intersetoriais para promoção à saúde não havia efetivação dessas ${ }^{31}$.

Diante de tais fragilidades, dificuldades na efetivação do cuidado multiprofissional e o reconhecimento da falta de preparo para abordar a obesidade também foram referidas por profissionais atuantes na assistência ao indivíduo com obesidade na atenção básica do estado do Rio de Janeiro, incluindo a atitude negativa e a baixa adesão dos usuários a tratamentos ${ }^{32}$. As estratégias sugeridas por Burlandy et al. (2020), para que tais desafios sejam superados, correspondem à corresponsabilização entre profissional e usuário, valorização de elementos para além da perda ponderal e ao exercício da multiprofissionalidade ${ }^{32}$.

No âmbito dos outros pontos de atenção da RAS, quatro participantes verbalizaram que não conheciam como se dava o cuidado ao público com obesidade em outros serviços de saúde, outras três falaram em uma rede com dificuldades sem indicar problemas específicos. Entre as demais, a visão da contribuição da RAS ao enfrentamento da obesidade foi orientada por impressões sobre a organização da assistência e encaminhamentos à atenção especializada.

Algumas profissionais concordaram que havia facilidade de encaminhar casos de obesidade para especialistas, outras expressaram que ao referenciar para o componente ambulatorial se deparavam com número reduzido de vagas, longas esperas até as datas agendadas e obstáculos no deslocamento do usuário até aos ambulatórios fora de seu território. Houve destaque também para a fragilidade na oferta de cuidado na prevenção e tratamento da obesidade na atenção básica, predominando ações fragmentadas e 
focadas nas comorbidades, com transferência para outros níveis da rede o cuidado com os casos mais graves de obesidade, havendo a percepção de maior resolutividade nesses casos.

\begin{abstract}
"Quando a gente referencia para um especialista já tem uma certa dificuldade de conseguir vaga, demora um certo tempo (...) às vezes até desestimula e não vai mais. Às vezes marca em um local que é distante para eles, diz que não conhecem, aí não vão também. Tem tudo isso". (P07, Auxiliar de Saúde Bucal)

"Nos outros níveis da rede tem bastante resolutividade os casos mais graves. (...) Eu acho que precisa mais trabalhos em atenção primária, porque $e ́$ um trabalho onde a promoção $e$ prevenção é muito e a mudança no estilo de vida é mais importante que qualquer outro recurso”. (P12, Médica) "O olhar que eu vejo é muito clínico. Você está com uma doença e por conta da obesidade você desenvolveu a hipertensão e você vai tomar isso, e se você desenvolver a diabetes vai tomar aquilo, você vai ser orientado para fazer as práticas, você vai ser encaminhado (...) esse paciente, ele precisa desse olhar clínico, mas ele precisa dos outros para gente ter uma efetiva mudança”. (P03, Enfermeira)
\end{abstract}

Embora tal prática seja pertinente ao fluxo dos usuários na RAS, o enfoque na referência a especialidades ambulatoriais não contempla todos os elementos de sua estrutura operacional, que prevê a atenção primária como centro de comunicação com pontos de atenção secundária e terciária, sistema de apoio diagnóstico e terapêutico, assistência farmacêutica, informação em saúde, logística e governança. Para além da estrutura operacional, o cuidado em rede propõe o envolvimento da comunidade e de equipamentos sociais. ${ }^{7}$

Assim como a interdisciplinaridade dentro das equipes de saúde é fundamental para um cuidado integral, a consolidação de iniciativas intersetoriais dentro dos territórios é estratégia elementar para que a obesidade seja enfrentada de maneira compatível a sua magnitude, em nível individual e coletivo, político e socioambiental, integrando ações de setores como saúde, educação, segurança e cultura. Consequentemente, o enfrentamento da obesidade a partir da ESF é premissa para consolidação de um efetivo cuidado em rede, por permitir diálogo contínuo com a comunidade, entre serviços de saúde e demais atores envolvidos na produção de cuidado.

\section{Considerações finais}

Este estudo evidenciou uma distância entre as concepções e construção de abordagens voltadas para a obesidade na ESF. Tal distanciamento aponta que a concepção e conhecimentos de profissionais valorizando a múltipla determinação da obesidade e a necessidade de ações interdisciplinares, contínuas e integradas não são suficientes para assegurar a implementação de tais características na prevenção e assistência terapêutica aos usuários nessa condição.

Nessa perspectiva, o cotidiano das equipes na atenção primária apresenta desafios na implementação de diretrizes fundamentais ao cuidado frente à obesidade, sobretudo à integralidade, autonomia, interdisciplinaridade, cuidado compartilhado e atuação em rede. Ainda, evidencia-se a necessidade de maior investimento na formação continuada dos profissionais da ESF, de forma associada à inserção de categorias profissionais de diferentes núcleos de conhecimento nesses espaços, visando o fortalecimento da interdisciplinaridade e da qualificação do cuidado para os usuários com obesidade. A educação permanente em saúde com valorização da integração de práticas e saberes de forma territorializada favorece vínculos e contextualização de práticas à perspectiva socioambiental, bem como à prevenção e manejo das comorbidades da condição obesa. 
Embora o enfrentamento da obesidade siga se consolidando na dimensão macropolítica por meio de publicações de referência, as práticas de cuidado ainda caminham pela tendência do modelo biomédico e curativista no cotidiano micropolítico, no qual a ESF é operacionalizada. Diante dos desafios observados, é necessário investir em ações de educação permanente em saúde que priorizem a temática da obesidade aos gestores e profissionais da ESF, e mediante à prática reflexiva reforcem o valor desse componente como potente dispositivo de vigilância alimentar e nutricional.

Além disso, fica evidente a necessidade de educação permanente para a desconstrução de preconceitos e atitudes negativas de profissionais frente ao indivíduo com obesidade. De igual modo, deve ser avaliada a articulação entre gestores, profissionais da ESF e comunidade para corresponsabilização na produção do cuidado, visando fortalecimento de vínculos, interdisciplinaridade, continuidade, e ações coletivas. A identificação de desafios intersetoriais reafirma que a obesidade não é um problema restrito ao setor de saúde, expondo a necessidade de iniciativas que dialoguem com todos os níveis de gestão, determinantes socioambientais e incorporação da visão sistêmica ao cotidiano da rede de atenção à saúde diante dessa epidemia.

\section{Referências}

1. Ministério da Saúde (Brasil). Vigitel Brasil 2018. Brasília: MS; 2019.

2. Wanderley EN, Ferreira VA. Obesidade: uma perspectiva plural. Cien Saude Colet 2010; 15(1): 185-194.

3. Associação Brasileira para o Estudo da Obesidade e da Síndrome Metabólica (ABESO). Diretrizes brasileiras de obesidade 2016. 4 ed. São Paulo; 2016.

4. Ministério da Saúde (Brasil). Estratégias para o cuidado da pessoa com doença crônica: obesidade. $\mathrm{CAB} \mathrm{n}^{\circ} 38$. Brasília: DAB/MS; 2014.

5. Cataneo C, Carvalho AMP, Galindo EMC. Obesidade e aspectos psicológicos: maturidade emocional, auto-conceito, locus de controle e ansiedade. Psicol. Reflex. Crit. 2005; 18(1): 39-46.

6. Dias PC, Henriques P, Anjos LA, Burlandy L. Obesidade e políticas públicas: concepções e estratégias adotadas pelo governo brasileiro. [periódico online] Cad. Saúde Pública 2017; 33(7): 00006016.

7. Brasil. Portaria GM/MS n ${ }^{\circ} 424$, de 19 de março de 2013. Redefine as diretrizes para a organização da prevenção e do tratamento do sobrepeso e obesidade como linha de cuidado prioritária da Rede de Atenção à Saúde das Pessoas com Doenças Crônicas. Diário Oficial da União; 2013.

8. Ministério da Saúde (Brasil). Portaria GM/MS n 2.436, de 21 de Setembro de 2017. Aprova a Política Nacional de Atenção Básica (PNAB), estabelecendo a revisão de diretrizes para a organização da atenção básica, no âmbito do Sistema Único de Saúde. Ministério da Saúde, Brasília - DF, 2017.

9. Ministério da Saúde (MS). Marco de Referência da Vigilância Alimentar e Nutricional na Atenção Básica. Brasília: DAB/MS; 2015.

10. Gomes CJG. Método de análise de conteúdo: ferramenta para a análise de dados qualitativos no campo da saúde. Rev Bras Enferm, 2004;57(5):611-4.

11. Campos GWS. A mediação entre conhecimento e práticas sociais: a racionalidade da tecnologia leve, da práxis e da arte. Cien Saude Colet, 2011; 16 (7):3033-3040.

12. Teixeira FV, Pais-Ribeiro JL; Maia ARPC. Uns desistem, outros insistem: semelhanças e diferenças no discurso de profissionais de saúde face à obesidade. Rev. Port. Sau. Pub. [periódico online] 2015; 33 (2): 137-147.

13. Bardin L. Análise de Conteúdo. $1^{\mathrm{a}}$ ed. São Paulo: Edições 70; 2011. 
14. Ricardi ML, Sousa MF. Educação permanente em alimentação e nutrição na estratégia saúde da família: encontros e desencontros em municípios brasileiros de grande porte. Cien Saude Colet, 2015; 20 (1)209-218.

15. Campos GWS. Saúde Pública E Saúde Coletiva: Campo e Núcleo de Saberes e Práticas. Sociedade e Cultura [periódico online] 2000; 3(1):51-74.

16. Campos GWS, Domitti AC. Apoio matricial e equipe de referência: uma metodologia para gestão do trabalho interdisciplinar em saúde. Cad. Saúde Pública, 2007, 23(2):399-407.

17. Araújo FK, Mourão GMJ, Costa MCB, Alberto NSLMC, Pereira RG, Ramos CV. Atenção nutricional para obesidade em unidades básicas de saúde. Revista Brasileira de Obesidade, Nutrição e Emagrecimento, 2019; 13 (79):385-393.

18. Lee B, Bartsch S, Mui Y, Haidari L, Spiker M, Gittelsohn J. A systems approach to obesity. Nutrition Reviews [periódico online], 2017; 75(supp11):94-106

19. Swimburn BA, Kraak VI, Allender S, Atkins VJ, Baker. The Global Syndemic of Obesity, Undernutrition, and Climate Change: The Lancet Commission report. 2019; $56 \mathrm{p}$

20. Palmeira CS, Garrido LMM, Lima ML, Guimarães AC, Ladeia AMT. Percepções acerca da condição de saúde de pessoas com obesidade. Rev Rene, 2016; 17 (5): 724-31.

21. Ministério da Saúde (Brasil). Vigitel Brasil 2016. Brasília: MS; 2017.

22. Gomes MF, Santos RSAF, Fontbonne A, Cesse EAP. Orientações sobre Alimentação ofertadas por Profissionais da Estratégia de Saúde da Família durante as Consultas aos Hipertensos e Diabéticos. Rev APS [periódico online], 2017; 20 (2): 203-211.

23. Almeida LM, Campos KFC, Randow R, Guerra, VA. Estratégias e desafios da gestão da Atenção Primária à saúde no controle e prevenção da obesidade. Rev. Gestão \& Saúde [periódico online], 2017; 8 (01):114-139.

24. Puhl R, Suhl Y. Health Consequences of Weight Stigma: Implications for Obesity Prevention and Treatment. Curr Obes [periódico online] 2015; 4: 182-190 (2015).

25. Rubino F, Puhl RM, Cummings DE, Eckel RH et al. Joint international consensus statement for ending stigma of obesity. Nat Med [periódico online] 2020; 13p.

26. Sonntag U et al. GPs' attitudes, objectives and barriers in counselling for obesity - a qualitative study. The European Journal of General Practice - Informa Health Care. [periódico online] 2012; 18 (1): 9-14.

27. Teixeira FV, Pais-Ribeiro JL; Maia ARPC. Crenças e práticas dos profissionais de saúde face à obesidade: uma revisão sistemática. Rev. Assoc. Med. Bras. [periódico online] 2012; 58 (2): 254262.

28. Camargo-Borges C, Japur M. Sobre a (não) adesão ao tratamento: ampliando sentidos do autocuidado. Texto contexto - enferm [periódico online]. 2008; 17(1): 64-71.

29. Klotz-Silva J, Prado SD, Seixas CM. A força do "hábito alimentar": referências conceituais para o campo da Alimentação e Nutrição. Physis. 2018; 27(4):1065-1085.

30. Farias DN, Ribeiro KSQS, Anjos UU, Brito GEG. Interdisciplinaridade e interprofissionalidade na estratégia saúde da família. Trab. Educ. Saúde. 2018; 16 (1): 141-162.

31. Silva KL, Sena R, Akerman MRR, Belga SMM, Rodrigues AT. Intersetorialidade, determinantes socioambientais e promoção da saúde. Ciência Cien Saude Colet. 2014;19(11):4361-4370.

32. Burlandy L, Teixeira MRM, Castro LMC, Cruz MCC et al. Modelos de assistência ao indivíduo com obesidade na atenção básica em saúde no Estado do Rio de Janeiro, Brasil. Cad. Saúde Pública [periódico on-line], 2020; 36 (3):e00093419

\section{Como citar este artigo:}

Figueiredo ATT, Tavares FCLP, Silveira PRRM, Costa EC, Oliveira AA, de Lira PIC. Percepções e práticas profissionais no cuidado da obesidade na estratégia saúde da família. Rev. Aten. Saúde. 2020; 18(64): 85-100. 